\title{
Learner Experiences and Perceptions of Using Social Media Tools in Formal Workplace Learning
}

\author{
Juha Leino, Erika Tanhua-Piiroinen, and Johanna Sommers-Piiroinen \\ The University of Tampere, School of Information Sciences/TRIM, Tampere, Finland \\ \{Juha. Leino, Erika. Tanhua-Piiroinen, \\ Johanna.Sommers-Piiroinen\} @uta.fi
}

\begin{abstract}
As social media tools are increasingly used in e-learning in the workplace, there is also a growing need for case studies to allow us to understand the underlying dynamics in order to develop best practices and to avoid potential pitfalls. Using qualitative approach, we studied a pilot training tailored for a large insurance organization that was conducted largely with chats, blogs, voice conferencing, and discussion forums. Our results show that despite challenges, social media use can produce value to e-learners and thus lend credence to many, so far weakly substantiated claims about their potential. In particular, synchronous tools enabled peer support, experience sharing, and networking. Still, the results also show that interactive learning process does not emerge automatically from adding social tools but needs to be designed and maintained. Based on learner views, we discuss how to improve the overall learning experience and make learning more efficient. Also, we discuss how to improve social media tools to better meet learner needs in e-learning in contrast to their recreational use.
\end{abstract}

Keywords: E-learning, continuing professional development, user studies.

\section{Introduction}

Social software is increasingly hailed as a way of integrating social learning-any "learning occurring within a group, an organization, or any social cluster" [14]—into e-learning [4], [12], as peer interaction in social context is thought to lead to effective construction of meaning and to better learning [3], [12]. Social media features are seen as offering educational affordances [2], [10], e.g. support for conversational interaction, social feedback, and social networking [10]. Such "sociability aspects" are seen as having "the most potential for enhancing education" [10]. Consequently, social media features are increasingly used in the education sector [2-4], [9].

At the same time, however, using social media features in the education sector is a new and still rather under-explored field [1], and so many papers today "uncritically ... rejoice in the possibilities of ... knowledge construction, critical reflective thinking and collaboration between learners, often without substantiating the enthusiasm" [2]. Without in-depth case studies [9], there is a clear and present danger of "misunderstandings, disappointments and irrelevant pedagogical practices" [13]. 
Such features are also making inroads into continuous professional development trainings and courses in the workplace [2], [4]. However, various ramifications of adding social media features to workplace trainings have been researched even to a smaller degree [1], [9]. Moreover, different perspectives in educational and workplace e-learning mean that we cannot necessarily apply results from one to another directly [1]. This results in a dire need for case studies - in particular, for "qualitative, studentcentered work" [6] — to uncover evidence-based best practices and potential pitfalls in designing and running workplace trainings enhanced with social media [1], [3], [9].

In this paper, we look at the perceptions and experiences of learners who took part in a pilot training that was tailored for one of the biggest insurance organizations in Finland. The tools used in the training were chat, voice conferencing, blog, and discussion forum. We used interviewing, questionnaires, and artifacts (e.g. blog texts) in addition to real-time observation of interactions (e.g. chats) and use logs to collect data. The data provides us with an in-depth look at the dynamics of what happens when social media features are used as the main approach in a workplace training. We found that despite many challenges, social e-learning can deliver on its promises, as learner interactions resulted in peer support, tip and experience sharing, and networking. Most value came out of social interactions facilitated with synchronous features.

In addition to describing social media tool use and related learner experiences and perceptions, we also discuss how to approach tackling some of the challenges that emerged in the training. We also discuss how to improve social media tools to better facilitate e-learning use, as goal-directed e-learning process (where people who may not know each other interact) needs different support than recreational use does.

\section{Background}

Social media is an umbrella term for mobile and web-based technologies that "create highly interactive platforms via which individuals and communities share, co-create, discuss, and modify ... content" [5]. Consequently, social media tools, e.g. wikis, blogs, and discussion forums, offer various educational affordances by e.g. facilitating and enabling sharing, communicating, and discovering information [10].

Using social media features in e-learning is part of the larger paradigm shift from teacher-oriented instruction to more student-centered learning. Learners are seen as active participants and co-produces-prosumers - instead of passive consumers and learning as a social process [10]. Consequently, what is learning and how it becomes visible needs to be re-evaluated [8]: Non-engaged learner may still be learning [7] and contributing does not automatically imply learning [8]. Learners may also fail to see social learning as real learning if it does not fit their pre-conception of learning [6].

Moreover, having social media features as part of the training does not guarantee that they are used properly_or at all—or that using them leads to learning [3], [10]. Without both learners and educators internalizing the new approach and its practices and attitudes that first need to be invented and designed, all-important mindset change and resulting behavioral change may fail to materialize [8], [10].

While reflective practices, e.g. writing blogs that others can see and comment instead of learning journals for instructor(s), are still part of social learning, social media in particular provide a community of peers [7] and thus enable getting "feedback, constructive criticism and validation" from interactions with peers [14]. 
This can function as a source of learning even if none of the learners knows the answer by enabling them to "contextualize current knowledge" and to develop "skills to deal with situations in the future" [7].

In social e-learning, increased social presence- "the degree to which a medium is perceived as conveying the presence" of the communicator [12]—is considered to increase learner satisfaction and both perceived and actual learning [1], [12]. Social presence is seen as having two dimensions, intimacy (interpersonal vs. mediated) and immediacy (synchronous vs. asynchronous) [12]. Social presence theory predicts that different media result in different levels of perceived intimacy and immediacy: Face to-face communication has the highest while asynchronous, technology-mediated communication forms have significantly lower levels of social presence [12].

Social media tools can be characterized by their immediacy as synchronous (e.g. chat and voice conference) or asynchronous (e.g. blog and discussion forum). Asynchronous tools (low feedback but high parallelism) suit information conveyance and reflective work while synchronous tools (high feedback but low parallelism) suit information convergence [12].

Using social media tools in e-learning requires new skills and this can create barriers to learning [1]. Learners with prior experience perceive using social media in learning as more satisfying and rewarding than those with little experience [12]. Importantly, social media skills acquired in other contexts, e.g. recreational use, may not translate directly into e-learning skills, as different contexts have their own use cultures [2].

\section{$3 \quad$ Method}

Using social media tools in workplace e-learning is a new field the studying of which necessitates qualitative case study approach that allows investigating complex social phenomena with a rich data set [9], [14]. Consequently, we used a qualitative approach to study adding social media tools to a formal workplace training, i.e. a training having "a structured program of instruction" leading to "a formal qualification or award," e.g. diploma or certificate, as opposed to informal learning, i.e. learning "acquired through everyday work and life" [11].

The two-month pilot training was provided by a professional training organization, Financial and Insurance Institute FINVA, and was tailored for internal trainers in one of the biggest finance and insurance organizations in Finland. The training had previously been taught through contact day lectures, and the role of electronic systems had basically been to allow returning assignments and downloading materials. Consequently, the training allowed us to explore the actual dynamics of adding social media features to a workplace training in real world, and our research goal was to uncover and describe the emerging dynamics and draw conclusions based on actual learner views and behavior rather than test any pre-described hypothesis.

Besides starting and ending days, the training was conducted entirely with social media tools: chat (three sessions), discussion forum (two assignments), blog (three blogs from ten given topics), and voice conferencing (two sessions). The forty employees taking the training were divided into seven small groups (5-6 learners per group) for chats and voice conferences but not for forums or blogs. As the organization has offices in about fifty cities, many learners logged in from different cities. 
As data of learner perceptions and experiences, we used chat contents (one chat topic was discussing the tools), blog contents (one of the given topics was to write about one's perceptions of learning with social tools), end survey data, and in-depth interviews of five learners. The semi-structured interviews were conducted at the end of the training by phone and were preceded by the end survey (where we solicited learners for interviews). The interviews were meant to and did provide further insight into survey answers. Table 1 summarizes the data we had on each small group. Apart from only four learners, we had at least one type of input from every learner.

Furthermore, we observed online interactions and learner behavior during interactions (e.g. chats) and through artifacts (e.g. forum postings) and log data (e.g. when postings were made). Consequently, we have a fairly comprehensive set of data of the training. However, at the same time, we have to exercise care in generalizing the results, as any case study inherently represents a specific set of circumstances. Consequently, our results represent more a starting point for further work than be-allend-all truths.

Table 1. Small groups by data: Numbers represent the number of learners in the small group of whom we had the particular type of material available (Blog and Chat refer to the tool-related topics mentioned above; No material refers to the number of small-group members from whom we had no survey, interview, blog (tool-related topic), or chat (tool-related topic) material available, i.e. the number of learners in the small group on whose views we have no material.)

\begin{tabular}{cccccccc}
\hline & $n$ & End survey & Interview & Blog & Chat & No material \\
\hline Small group 1 & 6 & 1 & 0 & 2 & 6 & 0 \\
Small group 2 & 6 & 3 & 1 & 2 & 4 & 1 \\
Small group 3 & 6 & 4 & 2 & 1 & 6 & 0 \\
Small group 4 & 6 & 4 & 0 & 2 & 3 & 1 \\
Small group 5 & 6 & 5 & 1 & 2 & 6 & 0 \\
Small group 6 & 5 & 2 & 1 & 3 & 5 & 0 \\
Small group 7 & 5 & 2 & 0 & 1 & 3 & 2 \\
\hline Altogether & $\mathbf{4 0}$ & $\mathbf{2 1}$ & $\mathbf{5}$ & $\mathbf{1 3}$ & $\mathbf{3 3}$ & $\mathbf{4}$ \\
\hline
\end{tabular}

The analysis approach was standard qualitative data coding approach. The materials in non-text format were first transcribed and then all material was coded into themes used in the in-depth interviews. Each theme was further divided into subcategories arising from the material, and some of the larger subcategories were further divided into sub-subcategories. While the end survey data provided us with some quantitative data, most of our data was qualitative.

\section{Synchronous Tools: Chat and Voice Conferencing Experiences}

The two synchronous features, chat and voice conferencing, were widely liked. They were seen as being close to face-to-face conversation and as providing a good amount 
of social presence: "... when it was real-time, it felt really nice. Even if the other person is in another city, it feels as if we're in the same room." Immediacy led to social presence also in chats: "...[we] got close. Like we were a chat family." Some learners said that synchronous tools enabled better "corridor conversations" than contact days by bringing more people and more focus into the exchange. Learning with synchronous tools was seen as more participatory and active than lecture-based learning.

While blog and forum texts were seen as something one produces "alone" and then submits, interactivity was seen as inherent in the synchronous features, as learners constantly reacted to each other: "....you compared your experiences to what others wrote or said..." This interactive process resulted in instantaneous and abundant feedback that gave learners "plenty of useful ideas."

Synchronous conversations were seen as flowing from "intuitive thinking," in part as the constant flow of interaction required quick responses, and as showing "how people see things," thus supporting experience sharing and peer support: "...the comments from others, feelings and experiences, they were the best..."

However, neither tool was without problems. Many learners felt that chats were too fast-paced: "... while you were writing something longer, others had moved to another topic." This led to overlapping of threads: "...many conversations [were] going on at the same time and it's hard to follow them all." As a result, following chats was challenging for many: "...everybody wrote at super-fast speed, commenting on different things. I was trying to keep up with it, scrolling up and down the message window..."

Voice conferencing in turn was plagued by turn-taking challenges. Learners found it difficult to know when they could talk: "... the problem was to know whose turn it was to talk-is the speaker still about to continue or is it proper to say something between..." and this could lead to some learners dominating the conversation: "...two people talk to each other and the four others listen in the background and maybe they would have good ideas to contribute but they end up forgetting them or the topic has moved on and the comment would now be out of place." Learners felt that moderation should be enhanced to handle such problems in chats and voice conferences.

Also, some learners felt that chats and voice conferences were light on substance"just chitchatting" - and suggested that there should be reading materials on which to base the conversations. In fact, while many felt that sharing experiences was one of the best things in the training, many others did not perceive it as learning: “...it should have been more professional. ...we were just sharing our own experiences. ... Although it's fun and all, there should have been some concrete teaching, too."

Some learners felt that the current group size (5-6 learners) was good while some felt that the groups were too big. Large groups made the pace too frantic for slower, "more deliberative" learners; smaller groups could help all learners be able to contribute more equally. Keeping groups small was also necessary to help everybody dare to participate: "...small enough to make learning efficient, because if the group is too large, people feel too shy or don't dare to participate or don't have time to talk..." 
Finally, using chat and voice conferencing at workplace brought its own challenges. Voice conferencing meant that learners had to watch what they said because other employees could hear them, thus impeding free flowing of conversation. Chats, on the other hand, were prone to interruptions, as the organization culture was to go to others to ask for help at any time; others simply did not realize that learners were busy. Still, chat as silent was seen as perhaps better suiting open office environments.

\section{Asynchronous Tools: Discussion Forum and Blog Experiences}

The two asynchronous tools used, blog and discussion forum, received a lukewarm reception. At best, they were found useful in that they forced one to slow down and reflect, but otherwise they were seen more as a chore. Postings in the asynchronous, non-real-time features were seen as something done "alone" and based on source materials as opposed to emerging from interactive process.

Learners consistently referred to blog and discussion forum texts as "assignments" and saw them also otherwise in terms of school assignments. Learners, perhaps consequently, felt that they had to submit "a well thought-out whole," "a finished article." Instead of "free association," blogs and forums produced "written articles."

Learners were not divided into small groups for blog and forum assignments. As most postings were made close to the deadline, learners faced a "gray mass" of text that discouraged them from reading and from commenting (not compulsory) them: “...I couldn't sacrifice so much working time ... people's postings were so long that you simply don't have time for it." For example, 70 blog postings (54\%) came in on the deadline day (March 18) and the three preceding days. The average length of a blog posting was about 1.3 pages (A4). Thus, within four days, learners received about 93 pages of material to read and to comment in one week-with more material flowing in after the deadline.

The situation was much the same for the forum assignments. Postings "flooded" in close to deadlines, resulting in them getting practically no comments and desultory readings at best: "...just too many messages, it numbs you and then you don't comment anything..." Neither did it help that all were writing on the same few topics: "... when there are you know a hundred postings about the same topic, nobody has the patience to read them all [laughed shortly]."

In fact, not using small groups in blog and forum assignments killed whatever chance there was for interactivity: "...[you can't] expect us to read ... 40 people's postings and comment them all... You should be able to concentrate on communicating between only a few people and exchange views that way." With smaller groups, interactive culture of sharing might have emerged: "...there might've been more discussion... It would have been more interactive."

Many learners experienced the resulting lack of feedback as discouraging: "...the assignments were rather massive ... but then you got no feedback at all on them. ... I started to wonder if it actually interested anybody at all." The situation was aggravated by some learners feeling that some others did the bare minimum but still got the same certificate. This, combined with the lack of feedback, 
made the more diligent learners question why they worked so hard, thus undermining their motivation. Consequently, one learner mentioned seeing no difference between posting answers to a blog and returning them by email while another one said that "Putting assignments in blogs felt a bit dumb, since I don't think anybody went there to read or comment them."

Many learners expressed hope that this solitary work would have been made more interactive. For example, one learner suggested that the long writings should have been submitted in smaller pieces so that others could have commented them (in small groups) and then the author could have continued based on the feedback.

Also, despite all the work that learners put into them, some learners felt that blog and forum posting contents were "superficial." Many saw them as a good source of ideas and knowledge that could be refined into something useful but not ready as such. Some learners suggested they should have tackled the postings as small groups: “...we never went through them properly. ...we would have refined them and turned the material into something that would have stayed as ... instructional material. Now it all stayed hanging in the air." Not only would this have resulted in more reusable materials, it would also have served to turn individual work into collective work. At the very least, learners would have read more texts with more focus.

\section{Discussion on the Implications of the Learner Experiences}

\subsection{Explicate Learning Goals and Benefits to Learners}

While many learners greatly appreciated sharing experiences and peer support, many others did not see this as real learning and missed a more theoretical approach. Some learners also expressed uncertainty about how to approach this kind of learning: "...we might have gotten a tad off the given topic [in chats] but I don't know if that's a good thing or a bad thing." Clearly, there is a need explicate to learners what learning with social media means, how learning takes place, and what is expected of them. Otherwise, some learners will feel confused: "...l was thematically lost ...the training was very different from what I expected and in that way I didn't get the benefit that I was expecting."

Naturally, explicating entails pointing out the benefits of the new approach. One benefit many learners appreciated was networking with other learners, including both making new acquaintances and improving ties with old ones: "Absolute the most fabulous thing about this training has been networking with other trainers. It gives you a certain peace of mind to know who to contact when you need help on something unfamiliar." The training also lowered the threshold in contacting: "It is also easier to contact these people in future when we already kind of know each other."

A related benefit is peer support: "It has been very gratifying to discuss various matters with my own small group and share one's sentiments." Training led people to emphasize each other: "You get a feeling that you are not alone with this thing but others are also in the same situation." Also, peers were 
seen to be in a good position to provide experience-based feedback, and hearing of "various experiences, thoughts, and practices" was felt to be a "significant" part of the training.

\subsection{Level Differences in Technical Abilities with Tool Training}

It is very dangerous to have expectations about the know-how level and technical skills of learners; e.g. even though Live Meeting was installed in everybody's computer as per company policy, not everybody knew how to use it. However, the training offered little tool training. Learners felt that this affected learning: "...it was a challenge to study the tool itself before you got to the actual matter."

Many learners felt that there should be a contact day focused on learning the tools to build confidence and to enable everybody to participate and contribute at equal footing. Learners felt that with proper tool training, they would, in fact, have saved precious time: "... what we would absolutely have needed was to go through the tools immediately at the beginning... but now we went in cold, and we have constant rush at work. ... it took way too much time to start figuring out how to use [the tools] during the working day." Also, learners needed training not just in technical use of tools but also in use culture related factors; e.g. not all learners knew how to approach generating content: "...blog was for me perhaps like, well, what I should write here, what I would talk about..."

Learners also emphasized tool-training because they saw social media tools as being part of the "modern" world and thus essential to know: "It's important to get to try different ways to study so that we could someday possibly utilize similar methods in our own trainings." Besides training purposes, learners were interested in social media tools because they saw in them a potential to support distance working and for getting just-in-time information from colleagues, for instance using chat to ask something while on the phone with a client. Now, however, some learners felt that training failed in teaching them tools: "It's still a mystery to me how it [Live Meeting] works." Some learners also expressed uncertainty about what they had learned: "I've learned to use social media in studying but do I know how to use it correctly? Maybe not."

Finally, some learners felt that using so many tools in the training had a fragmenting effect, especially since they were not familiar with the tools: "I'd have preferred concentrating on a fewer tools..." If learning tools is not in the locus of training, using fewer tools than here may be advisable.

\subsection{Learner Views on Interface Related Matters}

There were clear indications that learner needs for social media tool interfaces and features somewhat differ from the needs in recreational use. The reasons for differences appear related to various aspects of the use situation. In e-learning, the process is goal-oriented (the interaction has an agenda to fulfill), learners may not be familiar with each other but nevertheless need to interact at person-to-person level, and there is a cost-related pressure to have as big groups as possible. 
In chats, some learners wanted to have texts color-coded by the person. In e-learning, learners deal with people they may not be familiar with and learner-groups tend to be bigger. Consequently, identity awareness should be supported in the interface. If learners do not easily recognize each other and cannot get to know each other through the interface, no closeness develops and interaction stays at superficial level. The fragmentation of discussion into various threads in chats was also a problem for many learners. While recreational chatting can flow freely, e-learning process is goal oriented, and all information concerning an issue needs to stay connected. Thus, being able to tie different pieces of an issue thread together and being able to refer to earlier postings that may have disappeared from the current view need to be supported. Finally, many learners had difficulties writing fast enough and some worried about making typos. Predictive inputting and spell-checking could facilitate these problems. While there is a certain tolerance to typos in social media, e-learners in the workplace are nevertheless professional people who wish to make a professional impression on others.

In voice conferencing, learners had the same need as in chat to recognize the speaker with whom they may not be familiar and to get to know other learners. The interface should therefore support identity awareness. Also, there was a clear need to support turn-taking. Interfaces could support gesturing, turn-reserving and such e.g. through icons or other indicators. Moreover, being able to indicate agreement or disagreement in the interface-e.g. thumbs up or down-would allow learners to contribute to interaction without requiring a speaking turn.

In blogs and discussion forums, especially since the number of posting was high, there was a clear need to support learners to find postings that interested them. Many wished to read a particular person's postings or postings by their own small group. In social media, it is natural to allow sorting postings based on social aspects, such as authors and social connections between learners. Learners also wished to be able to have a summary, abstract or some other preview of a posting content to decide if they wanted to read it. While learners can be asked to write abstract to postings, there are also many automatic approaches that could be used, e.g. keyword clouds, that could also be used to help learners locate salient items.

\section{Conclusion}

Despite many problems evident in this pilot training, the learner perceptions and experiences lend credence to many claims about the potential of social e-learning. The interactive process, where it took place, resulted in experience sharing, abundant feedback, and peer support in addition to facilitating networking. That so much value materialized despite challenges further underlines the potential of social e-learning once the wrinkles have been smoothed out and best practices have been outlined.

However, the study also shows that benefits are not automatic. The social e-learning process has to be designed and maintained to make sure e.g. that group sizes suit the tools, the interactive process takes place, and learners have enough technical skills to focus on learning rather than on learning the tools.

Neither is social e-learning necessarily a be-all-end-all of workplace trainings. Now some learners found the learner-generated content somewhat lacking in 
substance. Consequently, it is necessary for future work to establish how best to use social e-learning for various types of learning goals. The implication from this study is that social e-learning works well when the knowledge exists within the learner community. However, when new, more theoretical matters are studied, it may be better utilized as supporting rather than as the sole approach to learning; e.g. contact days still appear to have their place, as some things, such as social media tools themselves, are best learned hands-on and face-to-face.

While more case studies are needed to understand how best to use social e-learning in workplace trainings, our results substantiate many promises and encourage further research.

\section{References}

1. Daneshgar, F., Van Toorn, C., Chan, S.C.E.: E-learning in Workplaces. In: ITI 6th Int. Conference on Information \& Communications Technology, pp. 65-70. IEEE (2008)

2. Dohn, N.B.: Teaching with Wikis and Blogs: Potentials and Pitfalls. In: 7th Int. Conference on Networked Learning, pp. 142-150 (2010)

3. Judd, T., Kennedy, G., Cropper, S.: Using Wikis for Collaborative Learning: Assessing Collaboration through Contribution. AJET 26(3), 341-354 (2010)

4. Kane, K., Robinson-Combre, J., Berge, Z.L.: Tapping into Social Networking: Collaborating Enhances both Knowledge Management and e-Learning. VINE 40(1), 62-70 (2010)

5. Kietzmann, J.H., Hermkens, K., McCarthy, I.P., Silvestre, B.S.: Social media? Get serious! Understanding the functional building blocks of social media. Business Horizons 54, 241-251 (2011)

6. Kirkpatrick, G.: Online 'Chat' Facilities as Pedagogic Tools: A Case Study. Active Learning in Higher Education 6, 145-159 (2005)

7. Ladyshewsky, R.K., Gardner, P.: Peer Assisted Learning and Blogging: A Strategy to Promote Reflective Practice during Clinical Fieldwork. AJET 24(3), 241-257 (2008)

8. Leino, J., Tanhua-Piiroinen, E., Sommers-Piiroinen, J.: Adding Social Media Features to Work-related Adult Learning: Educator Views, Expectations and Experiences. In: BIR 2011: Assoc. Workshops and Doctoral Consortium (Local Proc.), pp. 226-233. RTU (2011)

9. Lockyer, L., Patterson, J.: Integrating Social Networking Technologies in Education: A Case Study of a Formal Learning Environment. In: 8th IEEE Int. Conference on Advanced Learning Technologies, pp. 529-533. IEEE (2008)

10. McLoughlin, C., Lee, M.J.W.: Social Software and Participatory Learning: Pedagogical Choices with Technology Affordances in the Web 2.0 Era. In: Ascilite Singapore 2007, pp. 664-675 (2007)

11. Misko, J.: Combining Formal, Non-formal and Informal Learning for Workforce Skill Development. NCVER, Adelaide (2008)

12. Spencer, D.H., Hiltz, S.R.: A Field Study of Use of Synchronous Chat in Online Courses. In: Proc. of the 36th Hawaii Int. Conference on System Sciences, pp. 1-10. IEEE (2003)

13. Tynjälä, P., Häkkinen, P.: E-learning at work: theoretical underpinnings and pedagogical challenges. The Journal of Workplace Learning 17(5/6), 318-336 (2005)

14. Warne, L., Ali, I.M., Pascoe, C., Agostino, K.A.: Holistic Approach to Knowledge Management and Social Learning: Lessons Learnt from Military Headquarters. AJIS 9(1), 127-142 (2001) 